Invited Paper for the IMACS-International Symposium on Scientific Computing and Mathematical Modelling, Bangalore, India, December 7-11, 1992.

ANL/CP -75373

DE93 004189

\title{
BIASES IN MONTE CARLO EIGENVALUE CALCULATIONS*
}

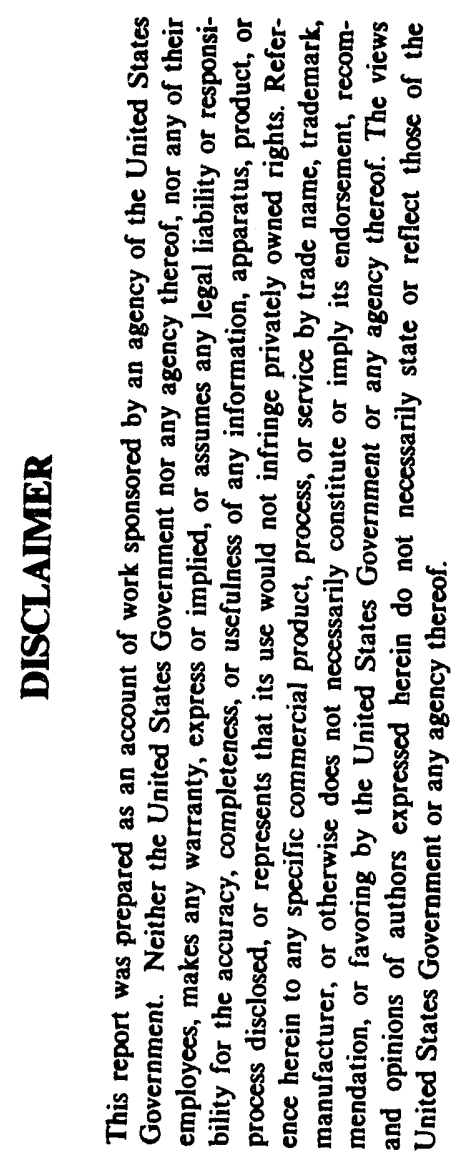

BY

Ely M. Gelbard

Argonne National Laboratory

Argonne, IL 60439

USA

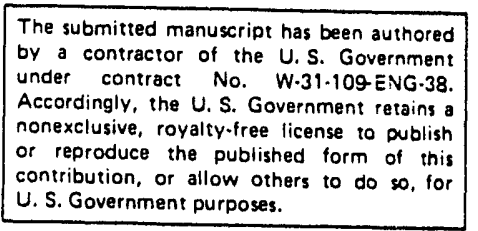

Work supported by the U.S. Department of Energy, Nuclear Energy Programs under Contract W-31-109-ENG-38. 


\title{
BIASES IN MONTE CARLO EIGENVALUE CALCULATIONS
}

\author{
Ely M. Gelbard \\ Argonne National Laboratory \\ Argonne, IL 60439 USA
}

\section{INTRODUCTION}

The Monte Carlo method has been used for many years to analyze the neutronics of nuclear reactors. In fact, as the power of computers has increased the importance of Monte Carlo in neutronics has also increased, until today this method plays a central role in reactor analysis and design.

Monte Carlo is used in neutronics for two somewhat different purposes, i.e., (a) to compute the distribution of neutrons in a given medium when the neutron source-density is specified, and (b) to compute the neutron distribution in a self-sustaining chain reaction, in which case the source is determined as the eigenvector of a certain linear operator. In (b), then, the source is not given, but must be computed.

In the first case (the "fixed-source" case) the Monte Carlo calculation is unbiased. That is to say that, if the calculation is repeated ("replicated") over and over, with independent random number sequences for each replica, then averages over all replicas will approach the correct neutron distribution as the number of replicas goes to infinity. Unfortunately, the computation is not unbiased in the second case, which we discuss here.

In the eigenvalue problem quantities of three types must be computed. First, one has to determine $k_{0}$, a single number such that, when all the reactor's fission cross sections are divided by $\mathrm{k}_{0}$, the reactor will be exactly critical. This means that the chain reaction will maintain itself so that the fission source-shape and the power level will remain unchanged indefinitely. It is $\mathrm{k}_{0}$ which plays the role of eigenvalue in the reactor eigenvalue calculation.

The second important computational result is the fission source-shape, and as the third result we take the distribution of all nuclear reaction rates excluding fission. It should be noted that the fission rate and the other nuclear reaction rates play very different roles in the eigenvalue calculation and must be treated differently in any theoretical analysis.

The eigenvalue calculation is performed by the power method, i.e.,

I. A fission source is assumed. This might be, for example, an analytically given function $S_{\mathrm{f}}^{0}$, of velocity, $\underline{\mathrm{v}}$, and position $\underline{\mathrm{r}}$, such that $S_{\mathrm{f}}^{0}(\mathrm{r}, \underline{\mathrm{v}}) \mathrm{d \underline {r }} \mathrm{d} \underline{\mathrm{v}}$ is the assumed number of fission neutrons in the phase-space volume $\mathrm{d} \underline{\mathrm{r}} \mathrm{d} \underline{\mathrm{v}} . \mathrm{S}_{\mathrm{f}}^{0}$ is normalized so that its phase-space integral is unity. 
II. $\quad S_{\mathrm{f}}^{0}$ is used as a probability density function (pdf) from which $\mathrm{M}$ sample starting neutrons (i.e., $M$ starting values of $\underline{r}$ and $\underline{v}$ ) are drawn.

III. For each starter one constructs a simulated history, using the known probabilities of the various events that a neutron might experience during its lifetime. This history is followed till the neutron is absorbed or completely escapes from the system under study.

IV. When a starter is absorbed it may create secondary fission neutrons. The expected number, $W_{f}$, of such neutrons, depends on the source-neutron's energy and position when absorbed. In an "analog" Monte Carlo one would record the absorption-site, $\underline{r}_{A}$, and the expected fission-weight $W_{f}$. After all starters are processed one has stored $M$ fission-sites and $M$ weights. In a nonanalog Monte Carlo, in contrast, one could take into account that an absorption, and subsequent fission, might occur whenever the neutron makes a collision in a mixture containing fissionable material. Correspondingly, at each collision site $r_{c}$ one would store the product $\mathrm{W}_{\mathrm{f}} \mathrm{x}(\mathrm{A} \mid \mathrm{C})$ where $\mathrm{P}(\mathrm{A} \mid \mathrm{C})$ is the conditional probability of absorption for a neutron colliding at $\underline{r}_{c}$, and $W_{f}$ is the expected number of offspring for an absorption at $\underline{r}_{c}$. In this case one might be left with more than $\mathrm{M}$ potential fission sites in storage.

V. In any case the sum of all weights stored at all sites is the first estimate, $k^{0}$, of the eigenvalue $k$; the weights divided by $k^{0}$ form a discrete pdf from which $M$ starting sites are chosen for the next source generation, etc.

VI. After a predetermined number, $\mathrm{N}$, of generations, the calculation terminates. The estimated eigenvalue is the mean of the $\mathrm{k}^{\mathrm{i}}$, s, averaged over all generations $i=0,1,2, \ldots, N$. As for the fission source-density, $S_{f}$, this obviously cannot be estimated at all space-points $\underline{r}$. Instead one estimates the volume-average of $S_{f}$ over each of a set of specified regions. This volume-average is estimated, in each region, as the sum, over all histories and all generations, of all fission weights deposited in the specified region, divided by the product NM.

It is generally assumed, on the basis of limited empirical evidence, that the biases are small, but more and better information about these biases is needed. A theoretical analysis of such biases has existed for a long time. ${ }^{(1)}$ Until recently, however, it produced almost nothing of practical value. Very early one could show that the magnitudes of biases are proportional to $1 / \mathrm{M}$; beyond this point very little more could be said.

Quite recently this situation has changed. (2) My main purpose here is to discuss some fairly new results which are now available, and to sketch work currently in progress. 


\section{BASIC THEORY}

The reactor eigenvalue problem may be written in the form $\mathrm{HU}_{0}=\mathrm{k}_{0} \mathrm{U}_{0}$. Here $\mathrm{H}$ is the operator which takes the $i$ 'th-generation fission source, $V^{i}$ (for any $i$ ), into the $i+1$ 'st source $\mathrm{V}^{\mathrm{i}+1}$, while $\mathrm{k}_{0}$ and $\mathrm{U}_{0}$ are, respectively, the eigenvalue of maximum modulus and the corresponding eigenvector. It is clear on physical grounds that if $V^{i}>0$, then $V^{i+1}>0$, i.e., that $\mathrm{H}$ is a positive operator. Most theoretical work on biases is based on the assumption that $\mathrm{H}$ has been discretized in some appropriate manner, so that $\mathrm{H}$ is a matrix. We suppose this to be true, and assume further that discretization has preserved the positivity of $\mathrm{H}$, so that $\mathrm{H}$ is a positive matrix. Thus, $\mathrm{k}_{0}$ is nondegenerate, and both $\mathrm{k}_{0}$ and $\underline{\mathrm{U}}_{0}$ are positive.

The theory underlying most U.S. work on biases is based on the following mathematical model of the Monte Carlo process:

$$
\begin{aligned}
& \underline{\mathrm{V}}^{\mathrm{i}+1}=\mathrm{H} \underline{\mathrm{V}}^{\mathrm{i} / \mathrm{k}^{\mathrm{i}}+\underline{\epsilon}^{\mathrm{i}}} \\
& \mathrm{k}^{\mathrm{i}}=\underline{\tau} \cdot \underline{\mathrm{V}}^{\mathrm{i}}, \underline{\tau}^{\mathrm{T}} \equiv(1,1,1, \ldots, 1) .
\end{aligned}
$$

Here $\epsilon^{i}$ is a stochastic error-vector. In fact the Monte Carlo process can always be represented as in Equ. (1) if $\underline{\epsilon}^{i}$ can be defined in any way we like. It becomes an approximation when one starts to specify the characteristics of $\underline{\epsilon}^{\mathrm{i}}$. For any reasonable Monte Carlo process we can expect that $E\left\{\underline{\epsilon}^{i}\right\}=0$, where $E$ denotes expected values. We come back to this point later.

Now let

$$
\underline{\mathrm{V}}^{\mathrm{i}}=\underline{\mathrm{MU}}_{0}+\underline{\delta}^{\mathrm{i}}
$$

with $\underline{\mathrm{U}}_{0}$ normalized so that $\underline{\tau} \cdot \underline{\mathrm{U}}_{0}=\mathrm{k}_{0}$.

Putting (3) into (1) we get

$$
\begin{aligned}
& \underline{\delta}^{i+1}=A \underline{\delta}^{i}-\frac{1}{M}\left(\underline{\tau} \cdot \underline{\delta}^{i} / k_{0}\right) A \underline{\delta}^{i}+\underline{\epsilon}^{i}, \\
& A \equiv\left(H / k_{0}\right)-\underline{U}_{0} \underline{\tau} / k_{0} .
\end{aligned}
$$

If the iterative process has become stationary (and we assume it will for large enough i) than

$$
\begin{aligned}
& E\{\underline{\delta}\}=-E\left\{\left(\underline{\tau} \cdot \underline{\delta^{i}} / k_{0}\right) A(I-A)^{-1} \underline{\delta}\right\} / M, \\
& \Delta \bar{k}=E\left\{\bar{k}-k_{0}\right\}, \bar{k} \equiv \frac{1}{N} \sum_{i=1}^{N} k^{i},
\end{aligned}
$$




$$
\Delta \underline{\bar{U}}=E\left\{\underline{\bar{U}}-\underline{U}_{0}\right\}, \underline{\bar{U}} \equiv \frac{1}{N} \sum_{i=1}^{N} \underline{U}^{i}
$$

where the $\Delta$ 's are biases. It will be seen that these biases are second-order in the error vectors $\underline{\epsilon}$.

Clearly the biases are due to statistical fluctuations in the $\mathrm{k}^{\mathrm{i}}$, the normalizing divisors in Equ. (1). It has been suggested that these biases can be substantially reduced by undoing the indicated normalizations. In effect, this is accomplished by the following procedure. ${ }^{(2)}$

Suppose we have carried out an ordinary power-method eigenvalue calculation. We have, then, constructed a sequence of eigenvector estimates $\underline{V}^{0}, \underline{V}^{1}, \underline{V}^{2}, \ldots, \underline{V}^{M}$. Let $k^{0}, k^{1}, k^{2}$, $\ldots, \mathrm{k}^{\mathrm{N}}$ be the corresponding power-method eigenvalue estimates. For some given (positive) constant $c$ define

$$
\begin{aligned}
& \underline{\tilde{V}}^{1}=\left(k^{0} / \mathrm{c}\right) \underline{\mathrm{V}}^{1}, \quad \underline{\mathrm{V}}^{2}=\left(\mathrm{k}^{0} / \mathrm{c}\right)\left(\mathrm{k}^{1 / \mathrm{c})} \underline{\mathrm{V}}^{2},\right. \\
& \underline{\tilde{\mathrm{V}}}^{i}=\left[\prod_{j=0}^{\mathrm{i}-1}\left(\mathrm{k}^{\mathrm{j} / \mathrm{c}}\right)\right] \underline{\mathrm{V}}^{i}, \mathrm{i} \geq 1 .
\end{aligned}
$$

Correspondingly

$$
\underline{V}^{i}=\left[\prod_{j=0}^{i-1}\left(c / k^{j}\right)\right] \tilde{\underline{v}}^{i}, i \geq 1
$$

From Equ. (10) and Equ. (1) we see that

$$
\begin{aligned}
& {\left[\prod_{j=0}^{i}\left(c / k^{j}\right)\right] \underline{\tilde{V}}^{i+1}=H\left[\prod_{j=0}^{i-1}\left(c / k^{j}\right)\right] \tilde{V}^{i} / k^{i}+\underline{\epsilon}^{i},} \\
& \underline{\tilde{V}}^{i+1}=H \tilde{V}^{i} / c+\underline{\tilde{\epsilon}}^{i}, \quad \underline{\tilde{\epsilon}}^{i} \equiv\left[\prod_{j=0}^{i}\left(k^{j} / c\right)\right] \underline{\epsilon}^{i} .
\end{aligned}
$$

Expanding the $\underline{\widetilde{V}}^{i}$ in eigenvectors of $H$ it will be seen that $E\left\{\widetilde{V}^{i}\right\}$ converges to a multiple of $\underline{U}_{0}$ as $i \rightarrow \infty$. Correspondingly the expected value of the mean of the $\underline{\tilde{V}}$ tends to a multiple of $\underline{U}_{0}$, i.e., the eigenvector estimate is unbiased. 
If $\mathrm{c}<\mathrm{k}_{0}$ the $\tilde{\widetilde{\mathrm{V}}}^{i}$ will tend to grow in magnitude exponentially with $\mathrm{i}$. The mean of the $\tilde{\tilde{V}}^{i}$ will then get contributions primarily from late iterates (with higher i). Early iterates will be wasted. Similariy for $c>k_{0}$ later iterates will be wasted. Thus it is desirable that $c \approx k_{0}$.

Suppose for simplicity that $c=k_{0}$. As earlier we write $\underline{\tilde{V}}^{i}=M \underline{U}_{0}+\underline{\delta}^{i}$, but now we find that

$$
\underline{\delta}^{i+1} \equiv \hat{\mathrm{H}} \widetilde{\delta}^{i}+\tilde{\epsilon}^{i}, \quad \hat{\mathrm{H}} \equiv \mathrm{H} / \mathrm{k}_{0}
$$

and we see that

$$
\underline{\delta}^{i}=\sum_{j=0}^{i} \hat{H}^{j} \underline{\tilde{\epsilon}}^{i-j-1}
$$

Because the largest eigenvalue of $\hat{\mathrm{H}}$ is unity the norm of $\hat{\mathrm{H}}^{j}$ does not tend to zero as $j \rightarrow \infty$. Therefore, the variance in $\widetilde{\delta}$ goes to infinity as $i$ increases. In the ordinary power method we find, in place of Equ. (13), that to first order in $\epsilon$

$-\quad \underline{\delta}^{i}=\sum_{j=0}^{i} A^{j} \underline{\epsilon}^{i-j-1}$.

But one can show that the eigenvalues of A are smaller than one in magnitude and it is for this reason that the variances in the $\underline{\delta}^{\mathrm{i}}$ are bounded. Thus, the same normalizing denominator which causes biases also reduces variance. Varients of the proposed method have been found to be useful under certain circumstances, but it seems fair to say that they do not eliminate the bias problem.

\section{BRISSENDEN-GARLICK RELATION FOR EIGENVALUE BIAS}

In 1986 the work of Brissenden ?.nd Garlick ${ }^{(2)}$ led to significant progress in the treatment of eigenvalue biases. This work specifically assumed an analog Monte Carlo scheme with an absorption estimator, exactly the "analog" algorithm described in Section I above. For our purposes here the key result of Ref. 2 is a simple expression for the eigenvalue bias, an expression which we will refer to as the B-G relation, and discuss later in some detail. More recently ${ }^{(3)}$ it has been shown the B-G relations is valid for a wide range of Monte Carlo algorithms. Below the B-G result is derived as in Ref. 2, but in a good deal more detail.

First it will be convenient to rewrite Equ.(5) in the form 
$\mathrm{E}\left\{\underline{\delta^{\mathrm{i}}}\right\}=-\mathrm{A}(\mathrm{I}-\mathrm{A})^{-1} \mathrm{~V} \underline{\hat{\underline{\tau}}} / \mathrm{M}, \quad \mathrm{V} \equiv \mathrm{E}\left\{\underline{\delta}^{\mathrm{i}}\left(\underline{\delta}^{\mathrm{i}}\right)^{\mathrm{T}}\right\}, \quad \underline{\hat{I}} \equiv \underline{\tau} / \mathrm{k}_{0}$.

Since $k^{i}=\underline{\tau} \cdot \underline{V^{i}} / M$,

$$
\Delta \mathrm{k}=\mathrm{E}\left\{\underline{\tau} \cdot \underline{\delta}^{i}\right\} / \mathrm{M}=-\mathrm{M}^{-2} \underline{\tau} \cdot \mathrm{A}(\mathrm{I}-\mathrm{A})^{-1} \mathrm{~V} \underline{\hat{I}}
$$

Next we consider the eigenvalue estimate

$$
\mathrm{K}=\mathrm{N}^{-1} \sum_{\mathrm{n}=1}^{\mathrm{N}} \mathrm{k}^{\mathrm{n}}
$$

The "real" variance in $\bar{k}$ (as opposed to the "apparent" variance defined below) is given by the expression

$$
\begin{aligned}
& \sigma_{\mathrm{R}}^{2}=[\sigma(\lambda)]^{2}=\mathrm{E}\left\{(\lambda)^{2}\right\}-[\mathrm{E}\{\bar{\lambda}\}]^{2}, \\
& \sigma_{\mathrm{R}}^{2}=\frac{1}{\mathrm{~N}^{2} \mathrm{M}^{2}} \sum_{\mathrm{i}=1}^{\mathrm{N}} \sum_{\mathrm{j}=1}^{\mathrm{N}} \underline{\tau} \cdot \mathrm{E}\left\{\underline{\mathrm{V}}^{\mathrm{i}}\left(\underline{\mathrm{V}}^{\mathrm{j}}\right)^{\mathrm{T}}-\underline{\mathrm{V}}^{\mathrm{i}}\left(\underline{\underline{V}}^{\mathrm{i}}\right)^{\mathrm{T}}\right\} \underline{\tau} .
\end{aligned}
$$

Setting $\underline{\mathrm{V}}^{\mathrm{i}}=\mathrm{M} \underline{\mathrm{U}}_{0}+\underline{\delta}^{\mathrm{i}}$ we find that

$$
\sigma_{\mathrm{R}}^{2}=(\mathrm{NM})^{-2} \sum_{\mathrm{i}, \mathrm{j}} \underline{\tau} \cdot \mathrm{E}\left\{\underline{\underline{\delta}}^{\mathrm{i}}\left(\underline{\delta}^{\mathrm{\delta}}\right)^{\mathrm{T}}\right\} \underline{\tau} .
$$

But $\underline{\delta}^{i+1} \approx \mathrm{A} \underline{\delta}^{\mathrm{i}}+\underline{\epsilon}^{\mathrm{i}}$, so that

$$
E\left\{\underline{\delta}^{i+1}\left(\delta^{i}\right)^{\mathrm{T}}\right\} \approx E\left\{\mathrm{~A} \underline{\delta}^{\mathrm{i}}\left(\underline{\delta^{i}}\right)^{\mathrm{T}}\right\}=\mathrm{AV}
$$

similarly,

$$
\begin{aligned}
& E\left\{\underline{j}^{j}\left(\underline{\delta}^{i}\right)^{T}\right\}=A^{j-i} V, j>i, \\
& E\left\{\underline{\delta}^{j}\left(\underline{\delta}^{i}\right)^{T}\right\}=V\left(A^{T}\right)^{i-j}, j<i .
\end{aligned}
$$

Here we have assumed throughout that $E\left\{\underline{\underline{i}}^{\mathrm{i}}\right\}=0$ for all $\underline{\delta}^{\mathrm{i}}$. Putting Equ. (23) and Equ. (24) into Equ. (21) we find that

$$
\sigma_{R}^{2}=(N M)^{-2} \underline{\tau} \cdot\left[\sum_{i=1}^{N} \mathrm{~V}+\sum_{i=1}^{N} \sum_{j=i+1}^{N} V\left(A^{T}\right)^{j-i}+\sum_{i=1}^{N} \sum_{j=1}^{i-1} A^{i-j} V\right] \underline{\tau},
$$


and approximately, for large enough $\mathbf{N}$,

$$
\sigma_{\mathrm{R}}^{2}=\left(\mathrm{NM}^{2}\right)^{-1} \underline{\tau} \cdot\left[\mathrm{V}+2(\mathrm{I}-\mathrm{A})^{-1} \mathrm{AV}\right] \underline{\tau} .
$$

The "apparent" variance, $\sigma_{\mathrm{AP}}^{2}$, is the variance in $\bar{k}$ computed as if the various $\mathrm{k}$ were statistically independent. In fact they are not, particularly in large reactors. If the distance which a neutron moves during its lifetime tends to be small compared to the reactor dimensions the fission-source shape will change only gradually from one generation to another. Thus, if i'th generation source neutrons are unduly concentrated in regions far from the reactor-core boundaries, this will also tend to be true for the (i+1)st generation, so that successive $k^{i}$ are positively correlated. Thus $\sigma_{\mathrm{AP}}^{2}<\sigma_{\mathrm{R}}^{2}$, and for very large reactors $\sigma_{\mathrm{AP}}^{2} \ll \sigma_{\mathrm{R}}^{2}$.

By definition

$$
\sigma_{\mathrm{AP}}^{2}+(\mathrm{N}-1)^{-1} \mathrm{E}\left\{\frac{1}{\mathrm{~N}} \sum_{\mathrm{i}=1}^{\mathrm{N}}\left(\tau \cdot \underline{\left.V^{i} / \mathrm{M}\right)^{2}}-\frac{1}{\mathrm{~N}^{2} \mathrm{M}^{2}}\left(\sum_{\mathrm{i}=1}^{\mathrm{N}} \underline{\tau} \cdot \underline{\mathrm{V}}^{\mathrm{i}}\right)^{2}\right\}\right.
$$

As in Ref. 2 we consider separately the two terms in Equ. (27). First

$$
\begin{aligned}
& -\frac{1}{N^{2}} E\left\{\sum_{i=1}^{N}\left(\underline{\tau} \cdot \underline{V}^{i} / M\right)^{2}\right\}=\frac{1}{N^{2}} \frac{1}{M^{2}} \sum_{i=1}^{N} E\left\{\underline{\tau} \cdot\left(M \underline{U}_{0}+\underline{\delta}^{i}\right)\left[M \underline{U}_{0}^{T}+\left(\underline{\delta}^{i}\right)^{T}\right] \cdot \underline{\tau}\right\}, \\
& \frac{1}{N^{2}} E\left\{\sum_{i=1}^{N}\left(\underline{\tau} \cdot \underline{V} \underline{i}^{i} M\right)^{2}\right\}=\frac{1}{N^{2}} \frac{1}{M^{2}} \sum_{i=1}^{N}\left(M^{2} k_{0}^{2}+2 M^{2} \Delta k+\underline{\tau} \cdot V \underline{\tau}\right) \text {, } \\
& \frac{1}{N^{2}} E\left\{\sum_{i=1}^{N}\left(\underline{\tau} \cdot \underline{V}^{i} / M\right)^{2}\right\}=\frac{1}{N}\left[k^{2}+\frac{\underline{\tau} \cdot V \underline{\tau}}{M^{2}}\right] \text {. }
\end{aligned}
$$

Here $\Delta \mathrm{k}$ is the eigenvalue bias and $\mathrm{k}$ is the biased Monte Carlo eigenvalue.

Next

$$
\begin{aligned}
\frac{1}{\mathrm{~N}^{3} \mathrm{M}^{2}} \mathrm{E}\left\{\left(\sum_{\mathrm{i}=1}^{\mathrm{N}} \underline{\tau} \cdot \underline{\mathrm{V}}^{\mathrm{i}}\right)^{2}\right\} & =\frac{1}{\mathrm{~N}^{3} \mathrm{M}^{2}} \sum_{\mathrm{i}=1}^{\mathrm{N}} \mathrm{E}\left\{\underline{\tau} \cdot \underline{\mathrm{V}}^{\mathrm{i}}\left(\underline{\mathrm{V}}^{\mathrm{j}}\right)^{\mathrm{T}}\right\} \\
& =\frac{1}{\mathrm{~N}} \sigma_{\mathrm{R}}^{2}+\frac{1}{\mathrm{~N}^{3} \mathrm{M}^{2}} \sum_{\mathrm{i}=1}^{\mathrm{N}} \sum_{\mathrm{j}=1}^{\mathrm{N}} \mathrm{E}\left\{\underline{\tau} \cdot \underline{\mathrm{V}}^{\mathrm{i}}\left(\underline{\mathrm{V}}^{\mathrm{j}}\right)^{\mathrm{T}}\right\},
\end{aligned}
$$




$$
\frac{1}{N^{3} M^{2}} E\left\{\left(\sum_{i=1}^{N} \underline{\tau} \cdot \underline{V}^{i}\right)^{2}\right\}=\frac{1}{N}\left(\sigma_{R}^{2}+k^{2}\right) .
$$

Thus

$$
\sigma_{\mathrm{AP}}^{2}=\frac{1}{\mathrm{~N}}\left(\frac{\underline{\tau} \cdot \mathrm{V} \underline{\tau}}{\mathrm{M}^{2}}-\sigma_{\mathrm{R}}^{2}\right)
$$

Finally

$$
\begin{aligned}
& \sigma_{\mathrm{R}}^{2}-\sigma_{\mathrm{AP}}^{2}=\frac{1}{\mathrm{NM}^{2}}\left[\underline{\tau} \cdot \mathrm{V} \underline{\underline{ }}+2 \underline{\tau} \cdot(\mathrm{I}-\mathrm{A})^{-1} \mathrm{AV} \underline{\underline{\tau}}\right]-\frac{1}{\mathrm{~N}}\left[\frac{\underline{\tau} \cdot \mathrm{V}_{\underline{\tau}}}{\mathrm{M}^{2}}-\sigma_{\mathrm{R}}^{2}\right], \\
& \sigma_{\mathrm{R}}^{2}-\sigma_{\mathrm{AP}}^{2}=\frac{1}{\mathrm{~N}}\left[\frac{2 \underline{\tau} \cdot(\mathrm{I}-\mathrm{A})^{-1} \mathrm{AV} \underline{\underline{\tau}}}{\mathrm{M}^{2}}+\sigma_{\mathrm{R}}^{2}\right] .
\end{aligned}
$$

It can easily be seen that the first quantity in the square brackets above is independent of $\mathrm{N}$ and $\mathrm{M}$, while the second is of order $1 / \mathrm{NM}$. Therefore,

$$
\sigma_{\mathrm{R}}^{2}-\sigma_{\mathrm{AP}}^{2} \approx \frac{2}{\mathrm{NM}^{2}} \underline{\tau} \cdot(\mathrm{I}-\mathrm{A})^{-1} \mathrm{AV} \underline{\tau}
$$

Comparing Eqs. (18) and (36) we see that

$$
-\Delta \mathrm{k}=\frac{\mathrm{N}}{2 \mathrm{k}_{0}}\left(\sigma_{\mathrm{R}}^{2}-\sigma_{\mathrm{AP}}^{2}\right)
$$

Equ. (37) is the B-G relation, but here derived for any Monte Carlo process correctly modelled by Eqs. (1) and (2). We have assumed only that $E\left\{\epsilon^{i}\right\}=0$ for any $\underline{V}^{i}$. But this must be true if, as is always required, the Monte Carlo process is unbiased for fixed-source calculations. Equ. (37) has been checked via test calculations, both for analog and nonanalog Monte Carlo methods. ${ }^{(3)}$ It agrees with empirical results very well in the analog case. In nonanalog calculations it agrees with test results within a standard deviation, but for the test problems statistical uncertainties are larger than in the analog case. More refined calculations are needed for a more conclusive test of Equ. (37) in nonanalog calculations.

But why this interest in the B-G relation? It should be understood that this relation is not simply a theoretical curiosity. It has, in fact, considerably practical value. ${ }^{(4)}$ One sees from Equ. (37) that 


$$
\begin{aligned}
& |\Delta \mathrm{k}|<\frac{\mathrm{N}}{2 \mathrm{k}_{0}} \sigma_{\mathrm{R}}^{2}, \\
& \left(|\Delta \mathrm{k}| / \sigma_{\mathrm{R}}\right)<\frac{\mathrm{N}}{2}\left(\sigma_{\mathrm{R}} / \mathrm{k}_{0}\right) .
\end{aligned}
$$

It will be seen that, if $\mathrm{k}$ is known to $.25 \%$ (i.e., $\sigma_{\mathrm{R}} / \mathrm{k}_{0}<.0025$, as is usually required for nuclear design work), and $\mathrm{N}<800$ (almost always true in practice) then $|\Delta \mathrm{k}|<\sigma_{\mathrm{R}}$. The bias is then smaller than the statistical uncertainty in $\mathrm{k}$, and in this sense is not significant.

\section{CURRENT WORK ON SOURCE-SHAPE BIASES}

In a sense then, eigenvalue biases (at least for standard reactor-design calculations) are no longer a live issue. It seems appropriate, therefore, to shift our attention to what is probably the most important, and perhaps also the easiest of the remaining bias problems, the problem of the fission-source bias.

Attempts to modify the B-G relation so as to cover the fission-source bias have so far not been successful. It is clear, for example, that the bias in the fission-source-average over some given space-region is not proportional to the difference between the true and apparent variance in this average. In a homogeneous medium with reflecting boundaries, region-averages of the fission source are not biased: but for a region covering any part (but not the whole) of the medium, $\sigma_{\mathrm{R}}^{2}>\sigma_{\mathrm{AP}}^{2}$.

At this point there is, however, another approach which seems worth pursuing, and that is to estimate the bias in the course of the Monte Carlo calculation, instead of bounding it analytically. To show how this might be done we go back to Equ. (4), which we write in the form

$$
\begin{aligned}
& E\left\{\underline{\delta}^{i}\right\}=-\frac{1}{M}(I-A) A E\left\{\delta_{k}^{i} \underline{\delta}^{i}\right\}, \delta_{k}^{i} \equiv k^{i}-k_{o} \\
& E\left\{\underline{\delta}^{i}\right\}=-\frac{1}{M} E\left\{\delta_{k}^{i} \sum_{n=1}^{\infty} A^{n} \underline{\delta}^{i}\right\} .
\end{aligned}
$$

But to leading order in $1 / \mathrm{M}$

$$
\begin{aligned}
& \delta^{i+1}=\mathrm{A} \delta^{i}+\underline{\epsilon}^{i}, A \underline{\delta}^{i}=\underline{\delta}^{i+1}-\epsilon^{i}, \\
& \mathrm{~A}^{2} \underline{\delta}^{i}=\mathrm{A} \delta^{i+1}-\mathrm{A} \epsilon^{i}=\delta^{i+2}-\underline{\epsilon}^{i+1}-\mathrm{A} \epsilon^{i}
\end{aligned}
$$




$$
\begin{aligned}
A^{n} \underline{\delta}^{i}= & \underline{\delta}^{i+n}-\sum_{m=0}^{n-1} A^{m} \underline{\epsilon}^{i+n-m-1}, \\
E\left\{\underline{\delta}^{i}\right\}= & -\frac{1}{M} E\left\{\delta \sum_{n=1}^{i} \underline{\delta}^{i+n}\right\} \\
& -\frac{1}{M} E\left\{\delta \delta_{k}^{i} \sum_{n=1}^{\infty} \sum_{m=0}^{n-1} A^{m} \underline{\epsilon}^{i+n-m-1}\right\} .
\end{aligned}
$$

In Equ. (40), the index $\ell=i+n-m-1 \geq i$. We have assumed that

$$
E\left\{\left(\underline{\epsilon}^{i} \mid \underline{\delta}^{i}\right)\right\}=0
$$

But if $\ell>$ i

$$
\begin{gathered}
\mathrm{E}\left\{\left(\underline{\epsilon}^{l} \mid \underline{\delta}^{\mathrm{i}}\right)\right\}=\int \mathrm{E}\left\{\left(\underline{\epsilon}^{l} \mid \underline{\delta}^{l}\right)\right\} \mathrm{P}\left(\underline{\delta}^{l} \mid \underline{\delta}^{l-1}\right) \cdot \mathrm{P}\left(\underline{\delta}^{l-1} \mid \underline{\delta}^{l-2}\right) \cdot \\
\ldots \mathrm{P}\left(\underline{\delta}^{\mathrm{i}+1} \mid \underline{\delta}^{\mathrm{i}}\right) \cdot \mathrm{d} \underline{\delta}^{l} \cdot \mathrm{d} \underline{\delta}^{l-1} \ldots \cdot \mathrm{d} \underline{\delta}^{\mathrm{i}+1}=0 .
\end{gathered}
$$

Therefore, to leading order in $1 / \mathrm{M}$,

$$
E\left\{\underline{\delta}^{i}\right\}=-\frac{1}{M} E\left\{\sum_{n=1}^{\infty} \delta_{k}^{i} \underline{\delta}^{i+n}\right\} .
$$

Thus, the bias in $\underline{V}^{i}$ is proportional to the sums of covariances between the eigenvalue fluctuation and fission source fluctuations in all later generations. It remains to be seen whether the necessary covariances can be computed accurately enough to give us practical estimates of the fission source bias. Of course, a similar method might be used to compute the eigenvalue bias also.

\section{CONCLUSIONS}

Obviously the rapid growth of the power of computers has had a decisive effect on the development of numerical techniques for every sort of computation. In particular, Monte Carlo methods have been strongly influenced by changes in computer speed and architecture. It would not be appropriate, here, to discuss the many ways that Monte Carlo has changed as computers have changed, and instead I will concentrate exclusively on new aspects of the Monte Carlo bias problem. 
Not so long ago it was customary to run Monte Carlo eigenvalue calculations with tens of thousands of histories. Now millions of histories are run, either on today's high-powered work stations (as long background calculations), or on mainframe computers like the various CRAYS. How does this change in capabilities affect the treatment of biases?

Consider, first, the bias in computed variances, a bias of a sort which has not been discussed here explicitly. It has already been noted that estimates computed in different generations are correlated. Normally this correlation is ignored and the apparent variance is used in place of the true variance. Sometimes the apparent variance is roughly corrected by the "MacMillan method." (5) But now an altogether different option is available. It may sometimes be desirable to run several replicas of an eigenvalue calculation, instead of putting all of the sample histories into a single replica. The true variance could then be estimated from the spread in results among replicas. Although this is not the most efficient strategy for running eigenvalue calculations, some loss in efficiency may, in some cases, now be an affordable luxury.

As for the eigenvalue bias, note first that small biases can now be computed directly in simple model problems where the true eigenvalue can be obtained deterministically. Such test calculations can tell us whether today's theoretical results are correct. Thus, we now have new motivation to develop realistic theories instead of simple rules-of-thumb.

Further, the eigenvalue bias can not only be bounded but, if necessary, can be computed from the true and apparent variances. This is, then, another reason for running multiple replicas.

Finally, it may now be possible to compute the covariances involved in the source-bias evaluation proposed above. If so we would have, for the first time, a practical tool for the estimation of source biases.

But it should be understood that much of what has ? nuclear reactor calculations, and is not necessarily true of "criticality safety" calculations. These are eigenvalue calculations for fuel being stored, reprocessed or transported. Characteristically the criticality safety calculation involves a set of large fuel lumps well separated and shielded from each other. In such configurations it may take many hundreds, even thousands of generations to establish a stable fundamental mode, and it is often difficult to determine whether the fundamental mode has been attained. Further, the final standard deviation may be much greater than $0.25 \%$. Under such circumstances the eigenvalue bias may be substantially larger than one standard deviation.

At any rate anomalous behavior has often been observed in criticality safety calculations, although there seems to be no reason why the theory discussed above should not hold in such Monte Carlo calculations. It is still not entirely clear what causes the observed anomalies. Some difficulties may be alleviated by the "superhistory" method of Ref. 2: but it seems fair to say that criticality safety calculations still have their own special mysteries. 


\section{REFERENCES}

1. E. M. Gelbard and R. E. Prael, "Monte Carlo Work at Argonne National Laboratory," ANL-75-2 (NEACRP-L-118), p. 202 (1974).

2. R. J. Brissenden and A. R. Garlick, "Biases in the Estimation of $k_{\text {eff }}$ and its Errors by Monte Carlo Methods," Ann. Nucl. Energy, 13, 63 (1986).

3. E. M. Gelbard, "Monte Carlo Biases, Generalization Beyond the Absorption Estimation," ANS Winter Mtg., San Francisco, CA, Trans. Am. Nucl. Soc., 64, p. 302 (1991).

4. E. M. Gelbard, "Present Status and Future Prospects of Neutronics Monte Carlo," Monte Carlo Conf. for Neutron and Photon Transport Calculations, Budapest, Hungary, Progress in Nuclear Energy, 24 \#1-3, p. 1.

5. D. B. MacMillan, "Monte Carlo Confidence Limi:s for Iterated-Source Calculations," Nucl. Sci. Eng. 50, p. 73 (1973). 

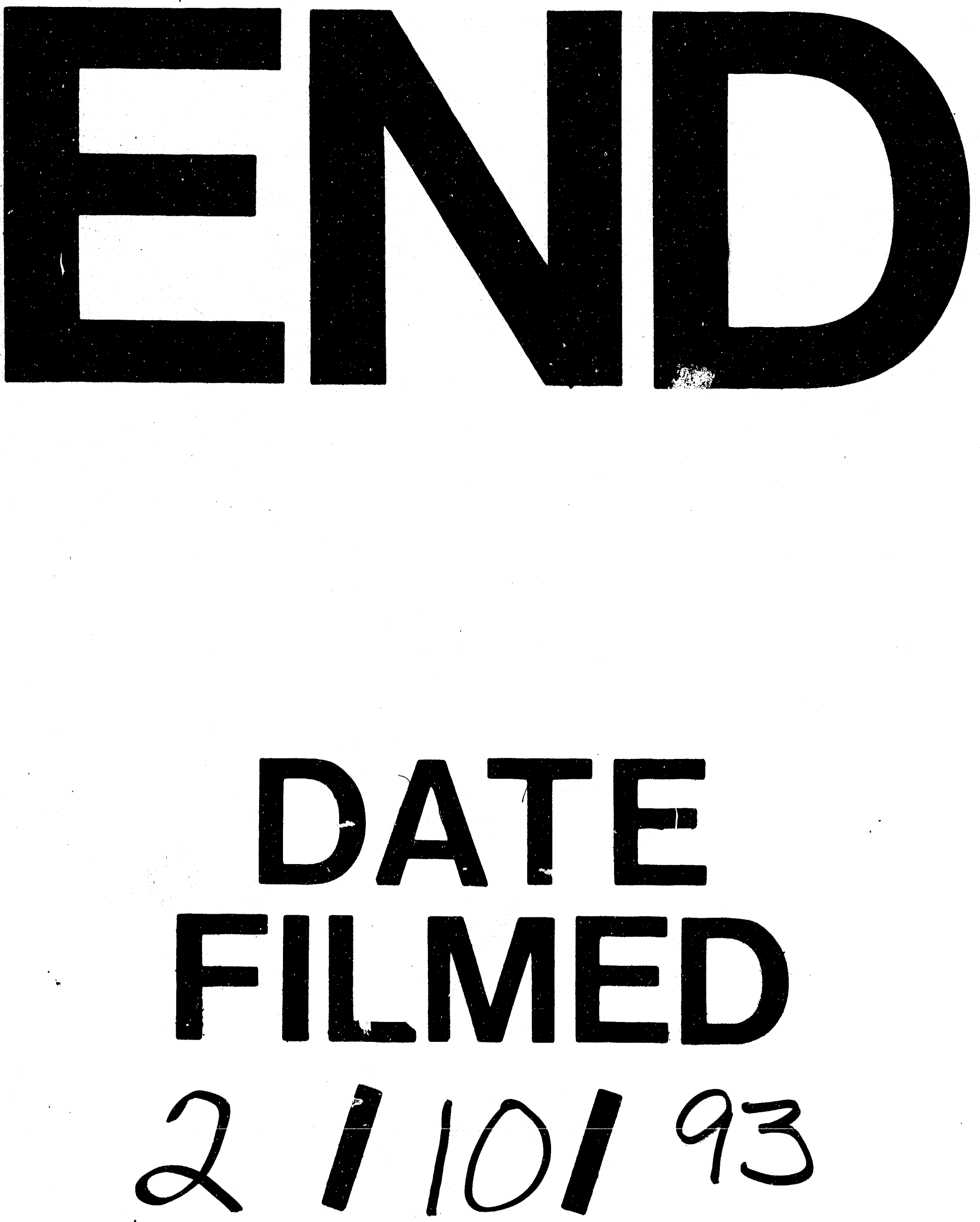

93 
